\title{
The Impact of Filter Settings on Morphology of Unipolar Fibrillation Potentials
}

\author{
Roeliene Starreveld ${ }^{1} \cdot$ Paul Knops $^{1}$ • Maarten Roos-Serote ${ }^{1} \cdot$ Charles Kik $^{2}$ - Ad J. J. C. Bogers ${ }^{2}$ • \\ Bianca J. J. M. Brundel ${ }^{3}$ - Natasja M. S. de Groot ${ }^{1}$
}

Received: 30 January 2020 / Accepted: 17 April 2020 / Published online: 14 May 2020

(C) The Author(s) 2020

\begin{abstract} identification of ablative targets.

Keywords Atrial fibrillation · High-resolution epicardial mapping $\cdot$ Electrogram morphology $\cdot$ Cardiac electrophysiology $\cdot$ Filter settings

$\begin{array}{ll}\text { DP } & \text { Double potential } \\ \text { FDT } & \text { Fractionation delay time } \\ \text { LA } & \text { Left atrium } \\ \text { LAT } & \text { Local activation time } \\ \text { PV } & \text { Pulmonary vein } \\ \text { PVL } & \text { Left pulmonary vein } \\ \text { PVR } & \text { Right pulmonary vein } \\ \text { RA } & \text { Right atrium } \\ \text { SNR } & \text { Signal-to-noise ratio } \\ \text { SP } & \text { Single potential }\end{array}$
\end{abstract}

Using unipolar atrial electrogram morphology as guidance for ablative therapy is regaining interest. Although standardly used in clinical practice during ablative therapy, the impact of filter settings on morphology of unipolar AF potentials is unknown. Thirty different filters were applied to 2,557,045 high-resolution epicardial AF potentials recorded from ten patients. Deflections with slope $\leq-0.05 \mathrm{mV} / \mathrm{ms}$ and amplitude $\geq 0.3 \mathrm{mV}$ were marked. High-pass filtering decreased the number of detected potentials, deflection amplitude, and percentage of fractionated potentials ( $\geq 2$ deflections) as well as fractionation delay time (FDT) and increased percentage of single potentials. Low-pass filtering decreased the number of potentials, percentage of fractionated potentials, whereas deflection amplitude, percentage of single potentials, and FDT increased. Notch filtering $(50 \mathrm{~Hz})$ decreased the number of potentials and deflection amplitude, whereas the percentage of complex fractionated potentials ( $\geq 3$ deflections) increased. Filtering significantly impacted morphology of unipolar fibrillation potentials, becoming a potential source of error in

\section{Abbreviations \\ AF Atrial fibrillation \\ BB Bachmann's Bundle \\ CFAE Complex fractionated atrial electrogram \\ CFP Complex fractionated potential}

Associate Editor Sunil Kapur oversaw the review of this article

Electronic supplementary material The online version of this article (https://doi.org/10.1007/s12265-020-10011-w) contains supplementary material, which is available to authorized users.

Natasja M. S. de Groot

n.m.s.degroot@erasmusmc.nl

1 Department of Cardiology, Erasmus Medical Center, Doctor Molewaterplein 40, 3015 GD Rotterdam, the Netherlands

2 Department of Cardiothoracic Surgery, Erasmus Medical Center, Rotterdam, the Netherlands

3 Department of Physiology, Amsterdam UMC, Vrije Universiteit Amsterdam, Amsterdam Cardiovascular Sciences, Amsterdam, the Netherlands

\section{Introduction}

Although insight into the pathophysiologic basis of fractionated atrial electrograms has increased in the past years, its "fact or artifact" remains a topic of debate. Extracellular electrograms - recorded directly from the heart - are generated by depolarization of cardiomyocytes, so signal morphology could provide information about the electrophysiological characteristics of the underlying myocardium. [1] Atrial potentials consisting of multiple components (i.e., fractionated) have been linked to abnormal conduction and arrhythmogenicity in patients with atrial fibrillation (AF) [1, 2], which led to targeted ablation of such fractioned potentials. However, the link between morphology of atrial potentials and pathology has proven to be anything but straightforward, 
considering that multiple physiological mechanisms and measurement properties, such as filtering, can cause fractionated potentials as well $[3,4]$. In the 1980s, Waxman and Sung discovered the phenomenon of frequency-dependent fractionation in human bipolar ventricular electrograms [5]. Klitzner and Stevenson showed that increasing the high-pass filter frequency above $10 \mathrm{~Hz}$ decreased potential duration and amplitude, whereas low-pass filtering altered potential amplitude slightly if decreased up to $100 \mathrm{~Hz}$ [6]. Such low- and highpass filters are commonly used in clinical practice, as well as utilization of a $50 \mathrm{~Hz}$ (or $60 \mathrm{~Hz}$ ) notch filter to suppress power-line interference.

Despite the clinical failure of targeting complex fractionated atrial electrograms (CFAEs) as stand-alone therapy [7-9], using atrial electrogram morphology as guidance for ablative therapy is regaining interest [10]. Up till recently, most clinically used ablative systems preferred bipolar above unipolar measurements, given its ability to reduce far-field potentials [11]. However, bipolar signals fail to represent incoherent waves during $\mathrm{AF}$, which is why currently emerging innovative ablative systems, such as RhythmView ${ }^{\mathrm{TM}}$ and AcQMap ${ }^{\circledR}$, prefer unipolar electrograms to identify local activation $[12,13]$. As the study of van der Does et al. demonstrated electrogram morphology at the epi- and endocardium to be comparable [14], epicardial electrograms are suitable to investigate signal morphology, particularly as direct contact between the electrode and atrial tissue can be assured.

To our knowledge, the impact of filtering on unipolar fibrillation potentials has never been investigated in humans. This study therefore aims to elucidate the consequences of high-pass, low-pass, and notch filtering on unipolar fibrillation potentials in AF patients.

\section{Methods}

\section{Study Population}

The study population consisted of ten adult patients with a history of paroxysmal or persistent AF undergoing elective open-heart mitral valve surgery in the Erasmus Medical Center Rotterdam. This study was approved by the institutional medical ethical committee (MEC 2010-054/MEC 2014393) $[15,16]$. Written informed consent was obtained from all patients. Patient characteristics (e.g., age, medical history, date of AF diagnosis) were obtained from the patient's file.

\section{Mapping Procedure}

Epicardial high-resolution mapping was performed prior to commencement to extra-corporal circulation, as previously described in detail [17-19]. A temporal bipolar epicardial pacemaker wire attached to the RA free wall served as a reference electrode. A steel wire fixed to the subcutaneous tissue of the thoracic cavity was used as an indifferent electrode. Epicardial mapping was performed with a 192electrode array (electrode diameter $0.45 \mathrm{~mm}$, interelectrode distances $2.0 \mathrm{~mm}$ ). The right pulmonary vein $(\mathrm{PV})$ area was mapped from the sinus transversus fold along the borders of the right pulmonary vein down towards the atrioventricular groove (as illustrated in the left panel of Fig. 1). Ten seconds of AF were recorded, including a surface ECG lead, a calibration signal of $2 \mathrm{mV}$ and $1000 \mathrm{~ms}$, a bipolar reference electrogram, and all unipolar epicardial electrograms. Data was stored on a hard disk after amplification (gain 1000), filtering (band-pass $0.5-400 \mathrm{~Hz})$, sampling $(1 \mathrm{kHz})$, and analog to digital conversion (16 bits).

\section{Filter Settings}

The impact of additional high-pass, low-pass, and notch filtering (i.e., narrow band-stop filter), as illustrated in the middle panel of Fig. 1, was investigated by changing filter settings one at a time, while keeping the others at default:

- High-pass filter: 0.5 (default), 1, 2, 3, 5, 10, 20, 30, 40, 50, $60,70,75,80,90,100 \mathrm{~Hz}$

- Low-pass filter: 400 (default), 300, 250, 200, 150, 100, $75,60,50,40,30,20,10 \mathrm{~Hz}$

- Notch filter at $50 \mathrm{~Hz}$ : off (default) and on

Settings were based on frequently used filter options within clinical mapping systems. Signals were zero-phase filtered with IIR Butterworth low- and high-pass filters (2nd-order: $12 \mathrm{~dB} /$ octave roll-off) and/or IIR notch filter with a quality factor of 30. Bode plots of the three filters are illustrated in ESM 1.

\section{Data Analysis}

Electrogram morphology was semi-automatically analyzed in custom-made Python 3.6 software. Deflections of atrial potentials were automatically marked if the slope was $\leq-0.05 \mathrm{mV} /$ $\mathrm{ms}$ and the amplitude $\geq 0.3 \mathrm{mV}$; the refractory period was set to $40 \mathrm{~ms}$ [20]. The steepest negative deflection of a potential was classified as the primary deflection and marked as the local activation time (LAT), whereas - in case of a fractionated potential - additional deflections were classified as secondary deflections. Electrograms with injury potentials and artifacts were excluded from analysis by manual assessment and consensus of two independent investigators.

For each different filter setting, peak-to-peak amplitude (voltage), fractionation ( $f$, number of deflections), and fractionation delay time (FDT, time interval between first and last deflection) of atrial potentials were analyzed. Peak-to-peak amplitude was analyzed for all deflections, as well as for 


\section{Epicardial mapping}

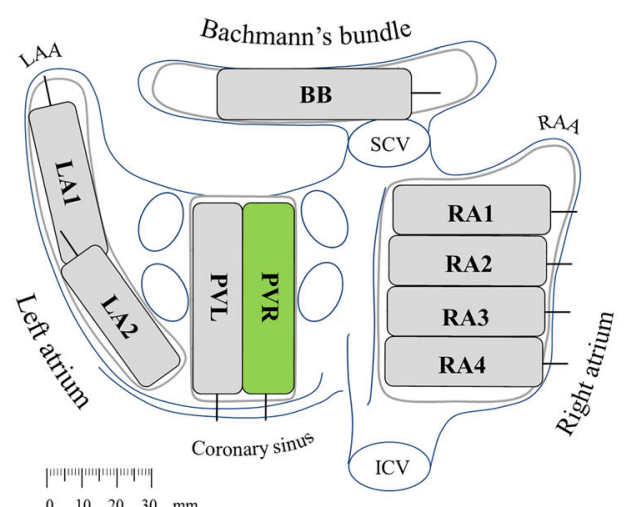

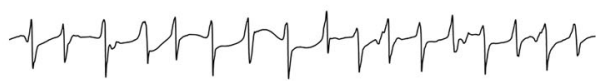

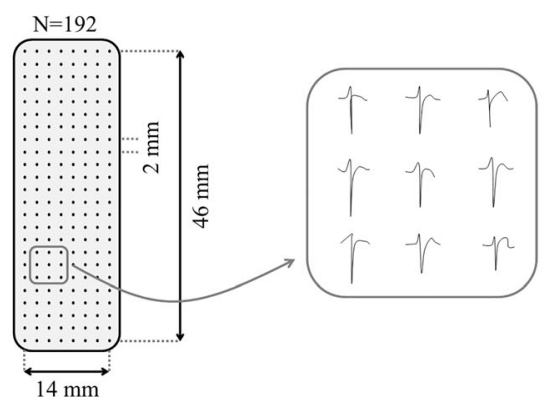

Filtering

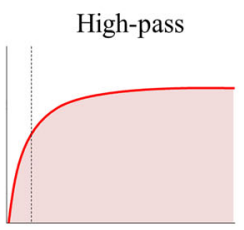

Low-pass

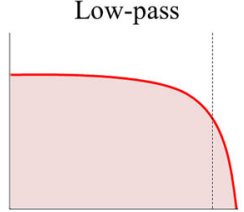

Band-pass
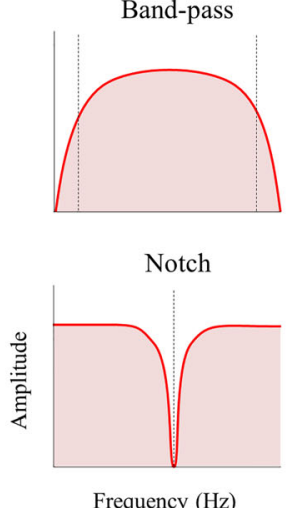

Morphology analysis
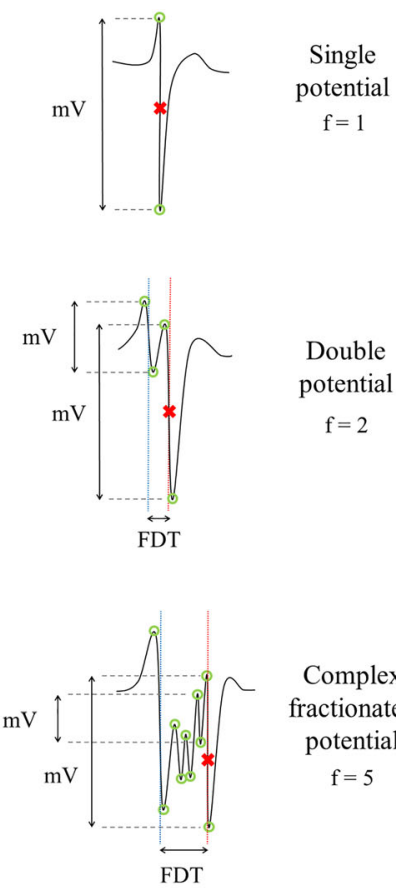

$\circ \quad \operatorname{Min} / \max$

* LAT
Fractionated potentials
Fig. 1 Schematic representation of mapping analysis. Left: the right pulmonary vein (PVR) area is mapped from the sinus transversus fold along the borders of the PVR down towards the atrioventricular groove. Using a 192-electrode array $10 \mathrm{~s}$ of AF is recorded. Middle: simplistic illustration of different filtering modes. Right: morphology analysis of all fibrillation potentials. The peak-to-peak amplitude of each deflection $(\mathrm{mV})$, fractionation (f, number of deflections), and fractionation delay time (FDT) is derived. Fibrillation potentials were classified as either

primary and secondary deflections separately. FDT was only derived for fractionated potentials $(f \geq 2)$. For each morphology parameter median values of all atrial potentials within the 192-array were derived and compared between filter settings. In addition, each fibrillation potential was classified as either single potential (SP, $f=1$ ), double potential (DP, $f=2$ ), or complex fractionated potential (CFP, $f \geq 3$ ). Figure 1 (right panel) illustrates derivation of morphology parameters and classification of fibrillation potentials.

\section{Statistical Analysis}

The impact of filtering on characteristics of unipolar fibrillation potentials, including amplitude, fractionation, and FDT, was analyzed using linear mixed-effect models, while accounting for clustered data within a patient. Analyses were done for the three different types of filtering separately: lowpass, high-pass, and notch filtering. The basic model only single potential $(f=1)$, double potential $(f=2)$, or complex fractionated potential $(f \geq 3)$. The steepest deflection of a potential is classified as the primary deflection (and marked as the local activation time), whereas additional deflections are classified as secondary deflections. BB, Bachmann's bundle; ICV, inferior caval vein; LA, left atrium; LAA, left atrial appendage; LAT, local activation time; PVL, left pulmonary vein; $\mathrm{RA}$, right atrium; RAA, right atrial appendage; $\mathrm{SCV}$, superior caval vein

included a random intercept and presumed no relation between filtering and morphology characteristics. Based on the Akaike Information Criterion it was checked whether addition of a random slope improved the model. To model any nonlinearity two splines were used. Residual plots were reviewed and $\log$ or square root transformed data was used if deviations from normality were observed. Statistical significance was tested using the likelihood ratio test. A $p$ value $<0.05$ was considered statistically significant. All statistical analyses were performed using R Statistical Software (RStudio, Inc., Boston, MA; version 1.0.153).

\section{Results}

Patient characteristics $(n=10)$ are shown in Table 1. All patients had a history of AF (paroxysmal $n=4$, persistent $n=6$ ) and age ranged from 56 to 77 years. In total, $3000 \mathrm{~s}$ of AF 
Table 1 Patient characteristics. $A F$ atrial fibrillation, $B M I$ body mass index, $F$ female, $I H D$ ischemic heart disease, $M$ male, $M V D$ mitral valve disease

\begin{tabular}{lllllll}
\hline Study ID & Underlying heart disease & Age (years) & Gender & BMI & Type of AF & $\begin{array}{l}\text { Time since AF } \\
\text { diagnosis (years) }\end{array}$ \\
\hline 1 & & & & & & 5.61 \\
2 & MVD & 70 & M & 25.3 & Paroxysmal & 2.07 \\
3 & MVD + IHD & 75 & M & 32.3 & Persistent & 0.25 \\
4 & MVD + IHD & 67 & M & 21.8 & Persistent & 20.33 \\
5 & MVD & 65 & M & 25.6 & Persistent & 0.72 \\
6 & MVD & 77 & F & 26.7 & Persistent & 1.13 \\
7 & MVD & 66 & M & 23.2 & Persistent & 0.61 \\
8 & MVD & 56 & M & 26.4 & Persistent & 0.06 \\
9 & MVD + IHD & 70 & M & 24.2 & Paroxysmal & 0.59 \\
10 & MVD & 64 & F & 34.6 & Paroxysmal & 0.28 \\
\hline
\end{tabular}

recordings were analyzed, consisting of 2,557,045 fibrillation potentials.

The impact of frequently used high-pass, low-pass, and notch filter settings on morphology of one example of a fractionated fibrillation potential is illustrated in Fig. 2. General consequences of all filter settings are discussed in the sections below.

\section{Impact of High-Pass Filtering on Morphology of Fibrillation Potentials}

Increasing the high-pass filter frequency had a negative impact on the number of detected fibrillation potentials and deflection amplitudes, as illustrated in Fig. 3. In the entire study population, the percentage of detected

\section{Default}

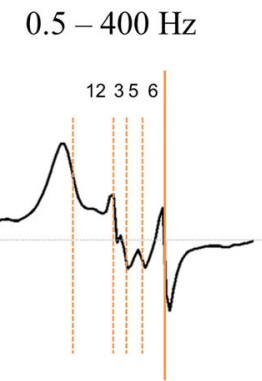

Notch

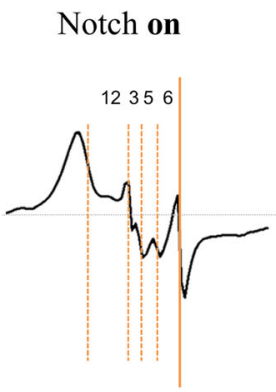

$1-400 \mathrm{~Hz}$
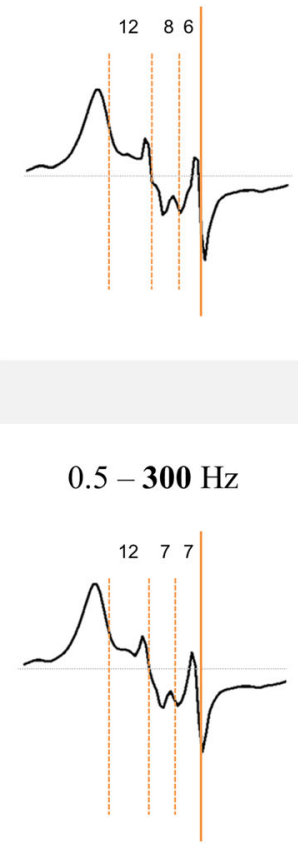

Fig. 2 Illustration of the impact of frequently used filter settings, i.e., high-pass, low-pass, and notch filtering, on morphology of one fractionated unipolar fibrillation potential. Detected deflections are

\section{High-pass filtering}
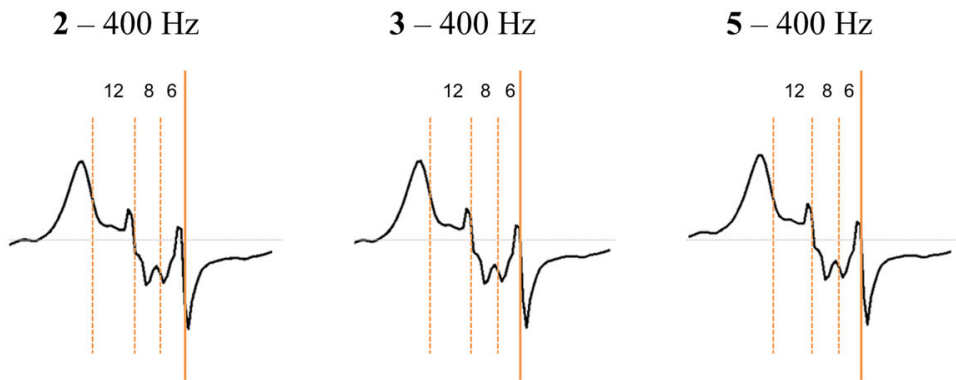

\section{Low-pass filtering}
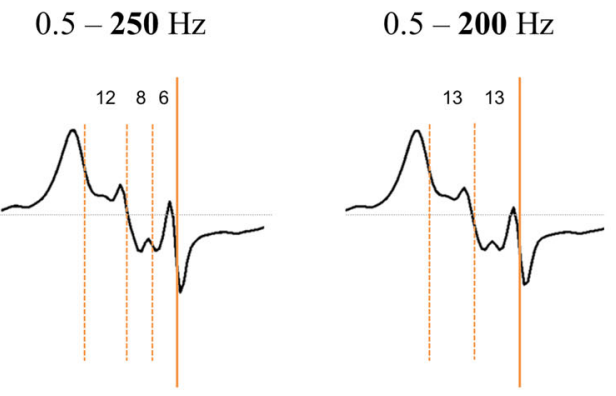

$0.5-100 \mathrm{~Hz}$

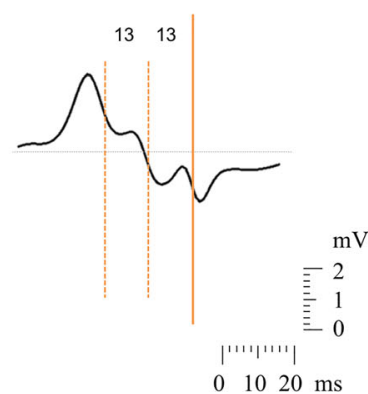

marked with orange vertical lines, a solid line representing the primary deflection and dashed lines the secondary deflections. The corresponding time interval between adjacent deflections is given (in $\mathrm{ms}$ ) 


\section{High-Pass Filtering}

Fibrillation Potentials

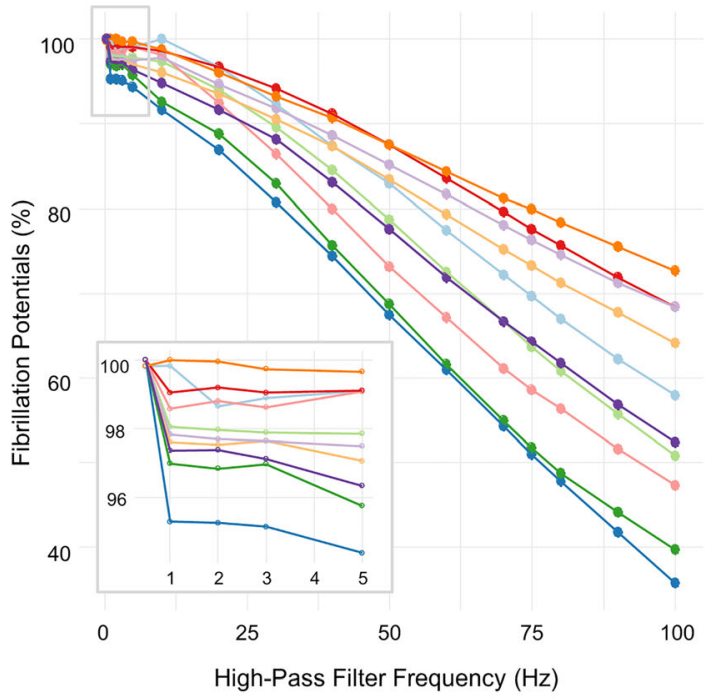

Primary Deflection Amplitude

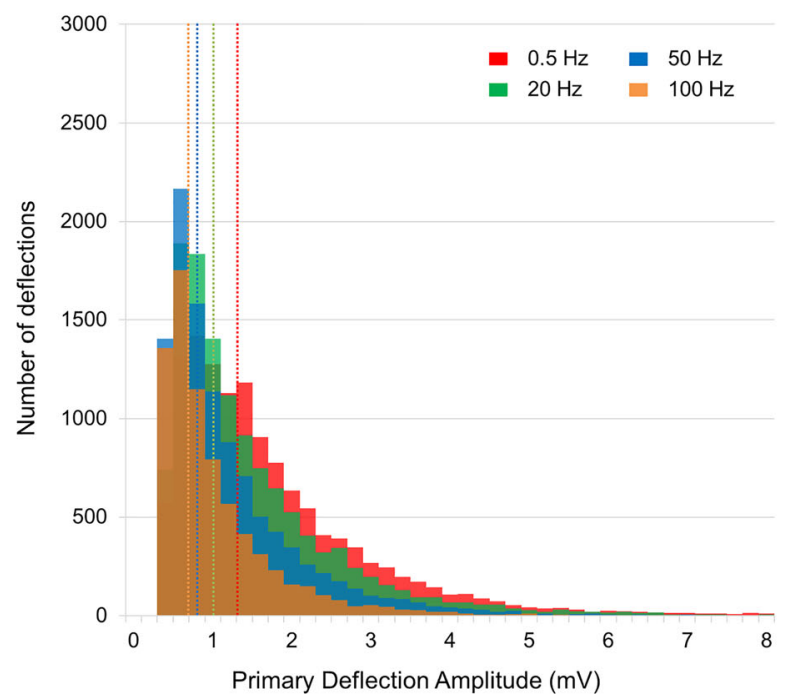

Fig. 3 The impact of high-pass filtering on detection of fibrillation potentials and deflection amplitude. Upper left: the number of detected fibrillation potentials, expressed as a percentage of maximal number of fibrillation potentials within the patient, of all patients. Upper right: overall median deflection amplitude of all patients. Lower left: stacked barplots of median primary deflection amplitude of one patient. Lower right:

fibrillation potentials gradually decreased, with a loss ranging from 27.2 to $74.5 \%$ at the maximum high-pass frequency of $100 \mathrm{~Hz}(p<0.01$, left upper panel). The overall median deflection amplitude decreased with increasing high-pass filtering for all patients (from 0.59$0.96 \mathrm{mV}$ to $0.44-0.57 \mathrm{mV}, p<0.01$, right upper panel).

\section{Deflection Amplitude}

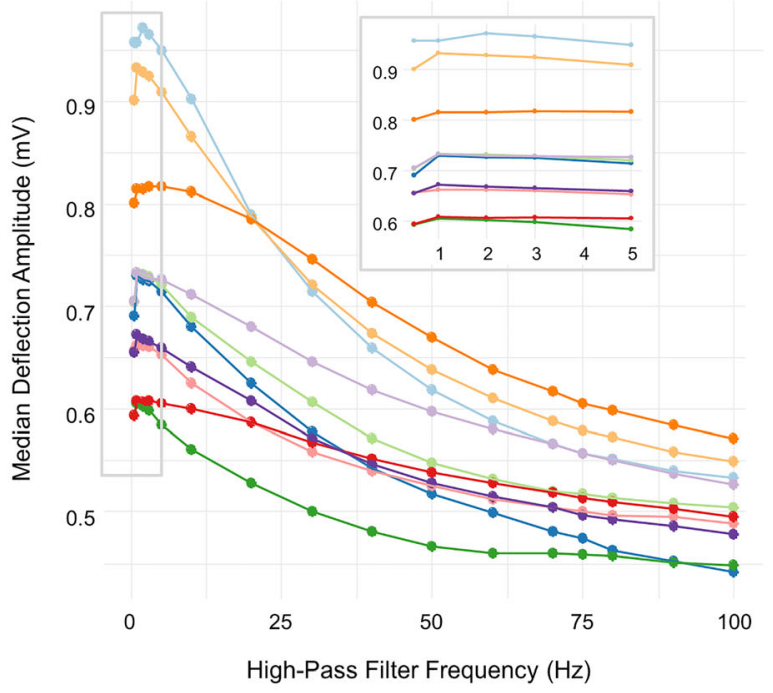

\section{Secondary Deflection Amplitude}

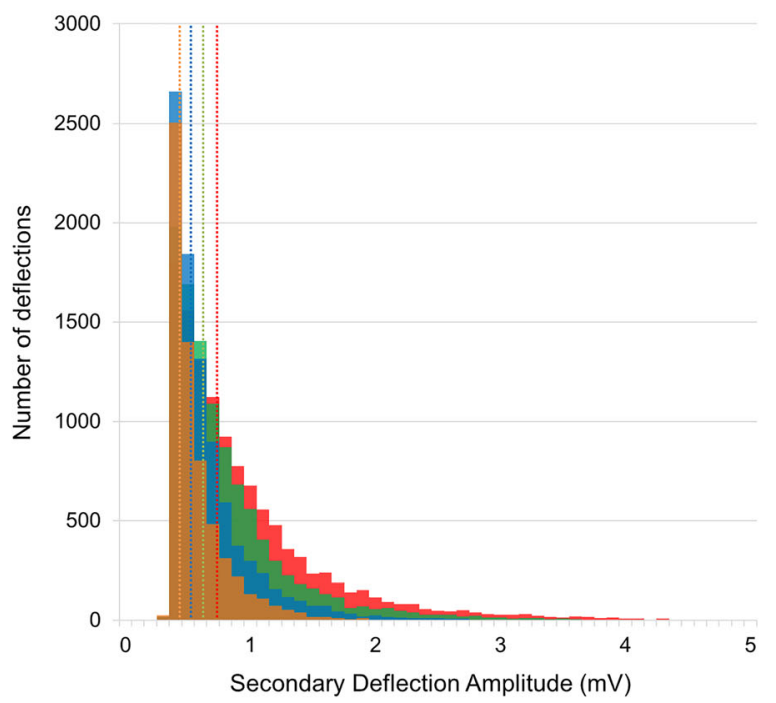

stacked bar-plots of median secondary deflection amplitude of one patient. For both lower figures, the data of patient 1 was taken as a representative case for all patients. The dotted vertical lines represent the median value of the corresponding stacked bar-plot, representing high-pass filtering at $0.5,20,50$, or $100 \mathrm{~Hz}$

This negative impact was also observed for median primary and secondary deflection amplitudes separately $(p<0.01)$, and was primarily caused by loss of high amplitude deflections, as illustrated in the histograms in the lower panel of Fig. 3 (obtained from one representative patient). 


\section{High-Pass Filtering}

\section{Fractionated Potentials}

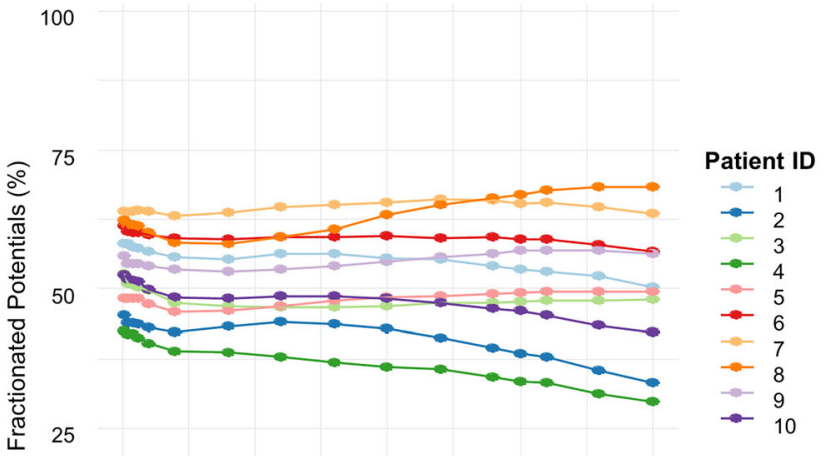

$\begin{array}{lllll}0 & 25 & 50 & 75 & 100\end{array}$

High-Pass Filter Frequency $(\mathrm{Hz})$

\section{Potential Type}

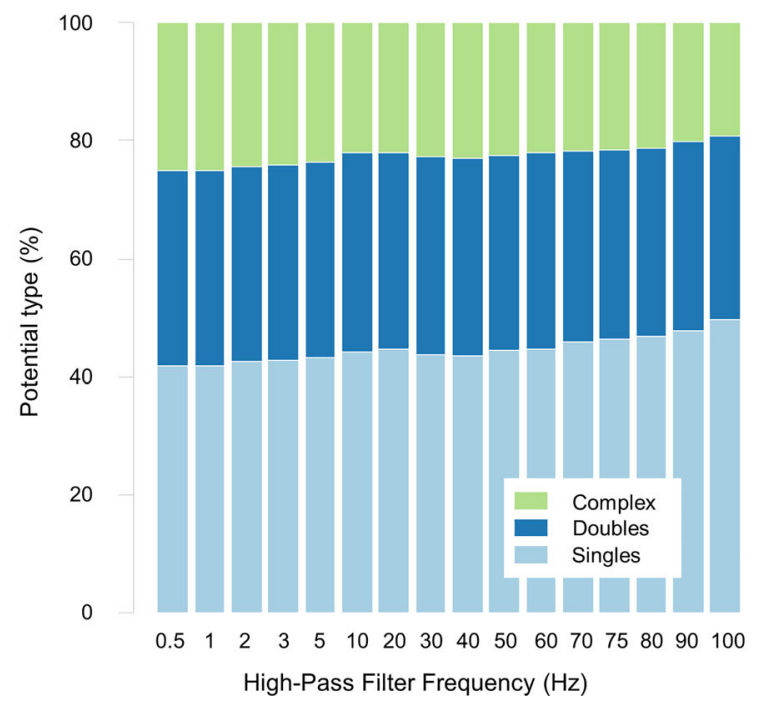

Fig. 4 The impact of high-pass filtering on fractionation. Upper left: the percentage fractionated potentials (two or more deflections per potential, expressed as a percentage of total detected fibrillation potentials) of all patients. Upper right: fractionation delay time (FDT, time interval between first and last deflection) of all patients. Lower left: stacked bar-plots of potential types obtained from one patient. Potential type is either single (one deflection), double (two deflections), or complex (three or more

The impact of high-pass filtering on the degree of fractionation, i.e., FDT and distribution of the different fibrillation potential types, is illustrated in Fig. 4. As observed in the left panel of Fig. 4, increasing the high-pass filter frequency from 0.5 to $100 \mathrm{~Hz}$ resulted in a slightly increasing percentage of SPs (from $36.1-57.6 \%$ to $31.6-70.2 \%$ ) at the cost of (primarily) CFPs (from $15.9-36.0 \%$ to $7.3-$

\section{Fractionation Delay}




\section{Impact of Low-Pass Filtering on Morphology of Fibrillation Potentials}

The impact of decreasing the low-pass filter frequency on morphology of unipolar fibrillation potentials is shown in Figs. 5 and 6. The left upper panel of Fig. 5 indicates an exponentially decreasing percentage of detected fibrillation potentials with decreasing the low-pass filter frequency $(p<0.01)$. As examples, low-pass filtering at $250 \mathrm{~Hz}$ induced a $2.5-11.6 \%$ loss of fibrillation potentials, which was $5.6-20.7 \%$ at $150 \mathrm{~Hz}$ and $20.3-58.4 \%$ at $75 \mathrm{~Hz}$. Decreasing the low-pass filter frequency exponentially increased the median deflection amplitude for all patients (from $0.59-0.96 \mathrm{mV}$ to $1.82-2.40 \mathrm{mV}$, right upper panel),

\section{Low-Pass Filtering}

\section{Fibrillation Potentials}

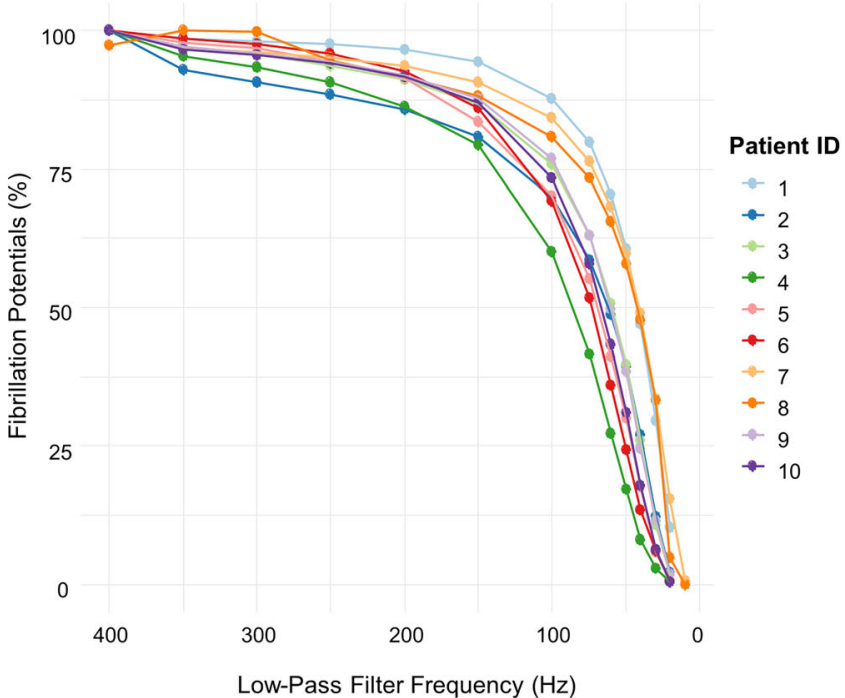

Primary Deflection Amplitude

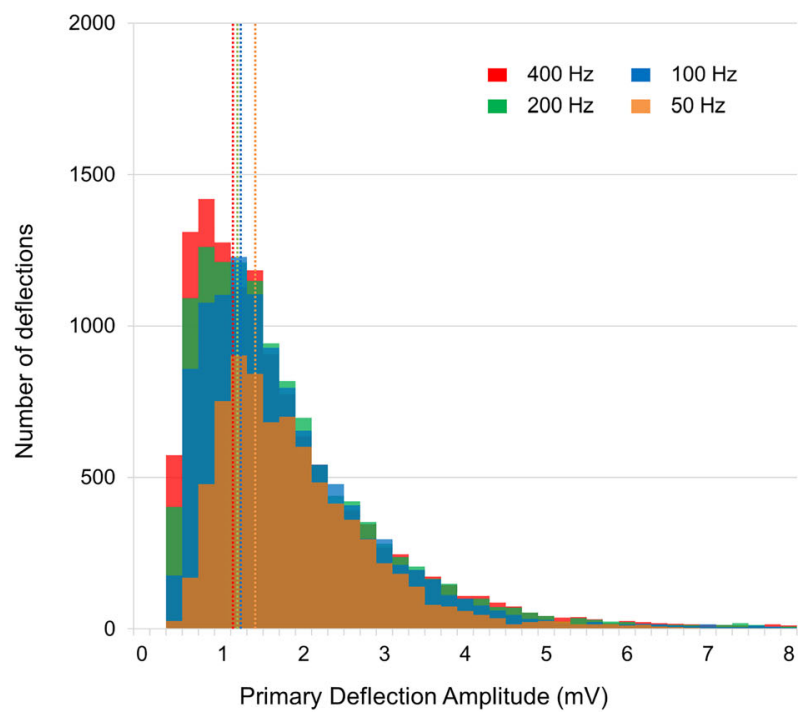

Fig. 5 The impact of low-pass filtering on detection of fibrillation potentials and deflection amplitude. Upper left: the number of detected fibrillation potentials (expressed as a percentage of maximal number of fibrillation potentials within the patient) of all patients. Upper right: overall median deflection amplitude of all patients. Lower left: stacked bar-plots of median primary deflection amplitude of one patient. Lower right:

\section{Deflection Amplitude}

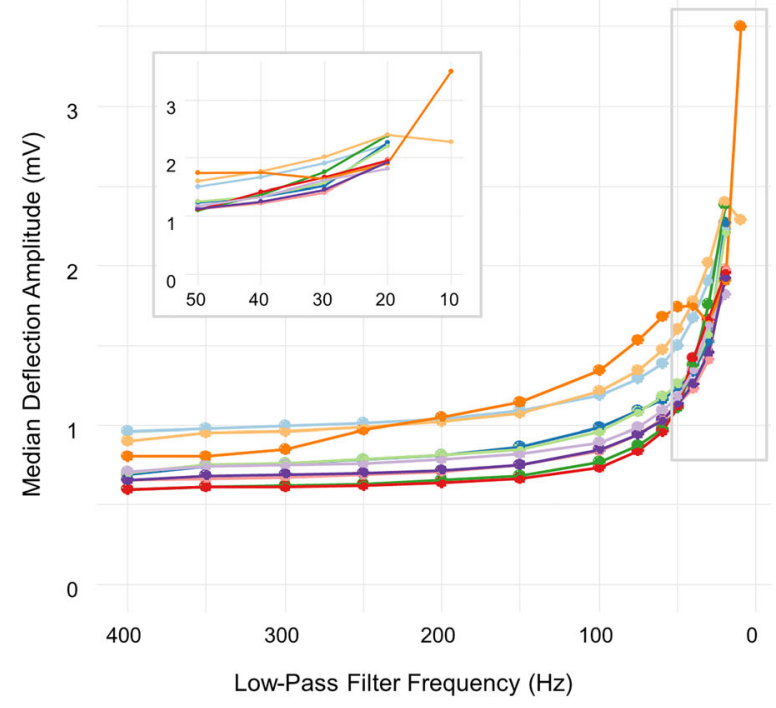

Secondary Deflection Amplitude

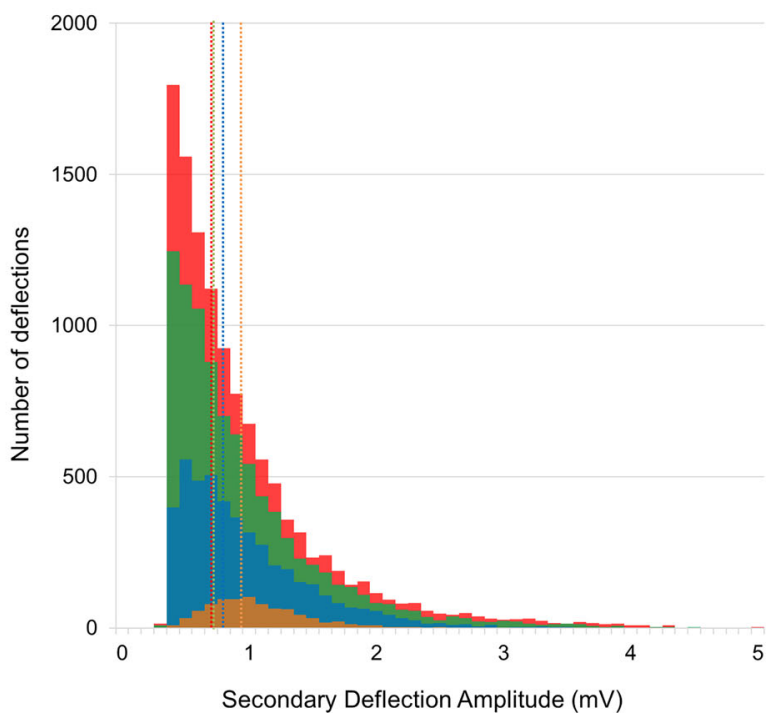

stacked bar-plots of median secondary deflection amplitude of one patient. For both lower figures, the data of patient 1 was taken as a representative case for all patients. The dotted vertical lines represent the median value of the corresponding stacked bar-plot, representing low-pass filtering at 400, 200, 100 or $50 \mathrm{~Hz}$ 
a trend that was also observed within primary and secondary deflections separately. This increase in deflection amplitude is primarily caused by a loss of low-amplitude deflections, as observed in the lower panel (histograms of one representative patient).

In Fig. 6, the impact of low-pass filtering on fractionation is visualized. The left upper panel illustrates a rapid decline of the percentage fractionated potentials $(p<0.01)$. Decreasing the low-pass filter frequency increased the percentage of SPs (from 36.1-57.6\% to $100 \%$ ) at the cost of both DPs (from $25.8-32.9 \%$ to $0 \%$ ) and CFPs (from $15.9-36.0 \%$ to $0 \%$ ). For all patients, the impact of low-pass filtering on DPs was mainly when filtering at $150 \mathrm{~Hz}$ or lower, whereas the presence of

\section{Low-Pass Filtering}

\section{Fractionated Potentials}

100

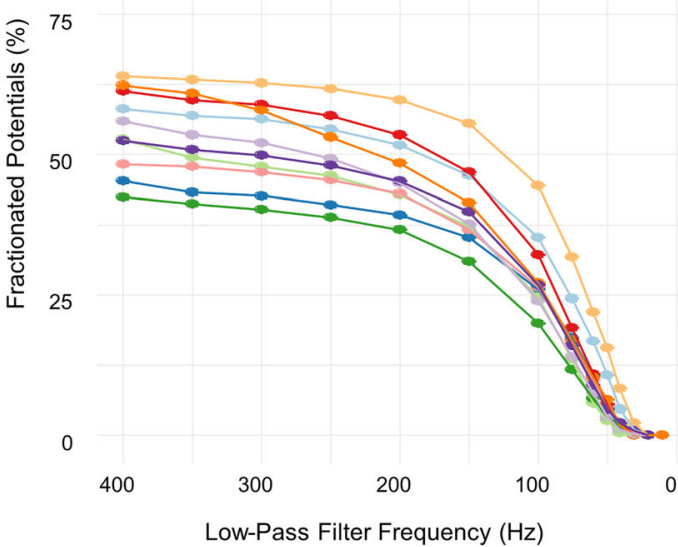

Type of Potential

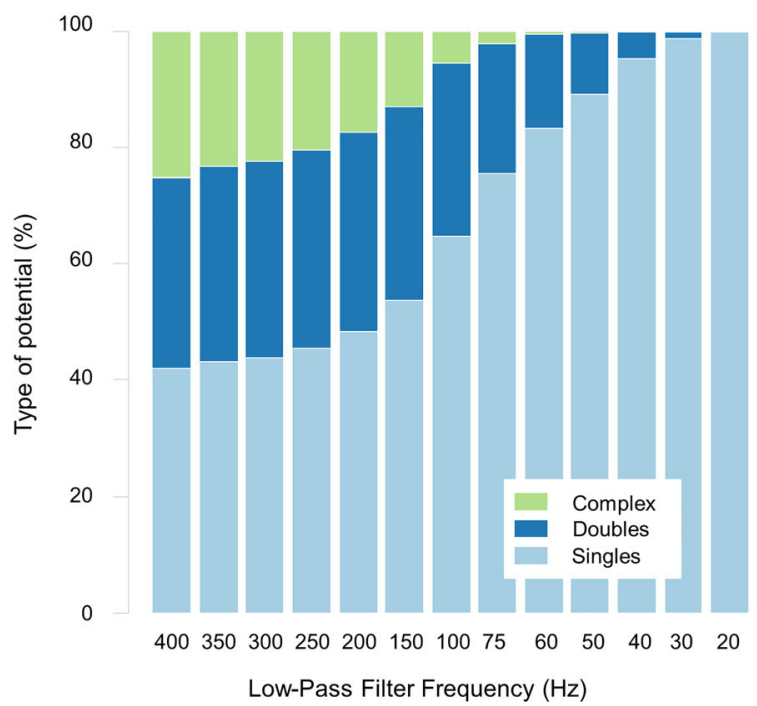

Fig. 6 The impact of low-pass filtering on fractionation. Upper left: the percentage fractionated potentials (two or more deflections per potential, expressed as a percentage of total detected fibrillation potentials) of all patients. Upper right: fractionation delay time (FDT, time interval between first and last deflection) of all patients. Lower left: stacked barplots with the distribution of potential types of one patient. Potential type is either single (one deflection), double (two deflections), or complex

\section{Fractionation Delay}

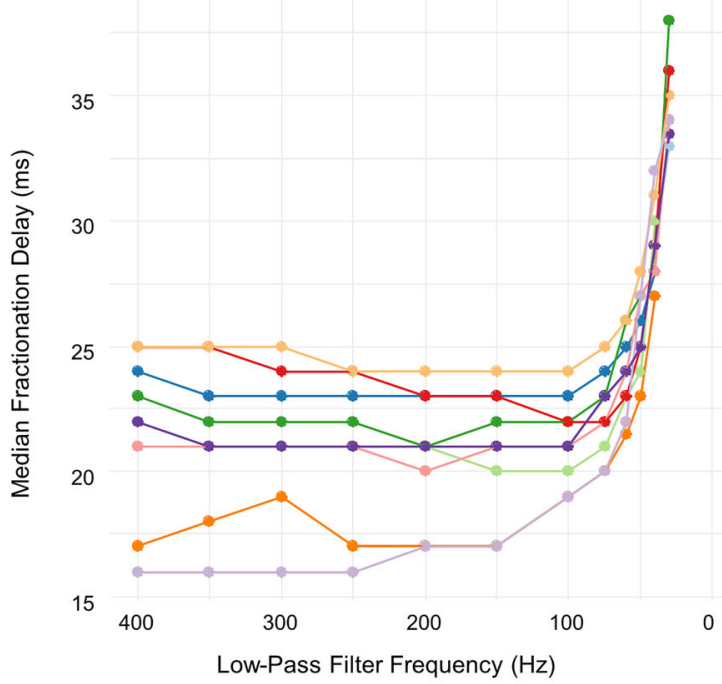

Fractionation Delay

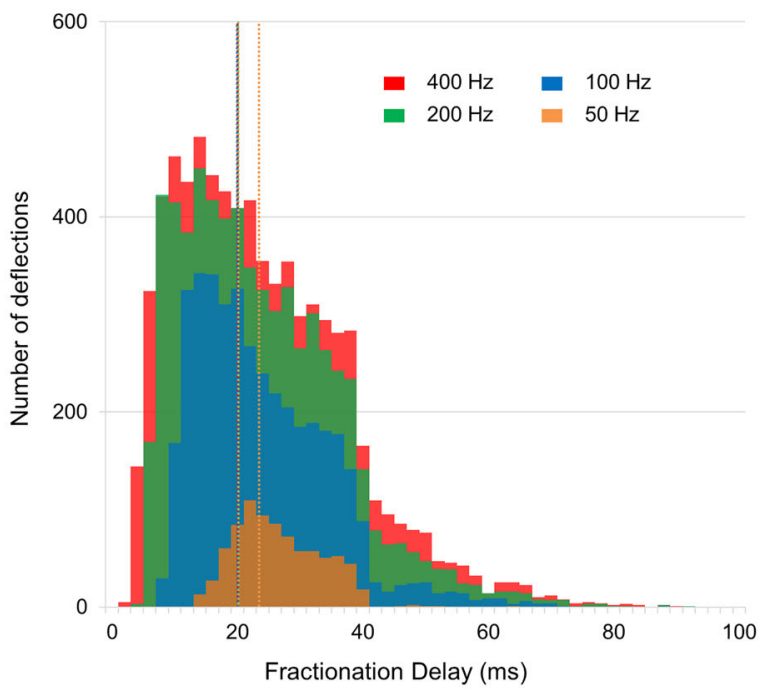

(three or more deflections). Distribution is expressed as a percentage of the total detected fibrillation potentials. Lower right: stacked bar-plots of median FDT of one patient. The dotted vertical lines represent the median value of the corresponding stacked bar-plot representing low-pass filtering at 400,200,100, or $50 \mathrm{~Hz}$. For both lower figures, the data of patient 1 was taken as a representative case for all patients 
CFPs diminished almost linearly at frequencies below $400 \mathrm{~Hz}$ until none were left (left lower panel, stacked bar-plot of one representative patient). Decreasing the low-pass filter frequency from 400 to $100 \mathrm{~Hz}$ did not substantially increase median FDT (from $16.0-25.0 \mathrm{~ms}$ to $19.0-24.0 \mathrm{~ms}$ respectively, right upper panel), since both fractionated potentials with a short and long FDT disappeared due to the filtering (right lower panel). Low-pass filtering below $100 \mathrm{~Hz}$ however resulted in a steep rise of FDT (up to $38.0 \mathrm{~ms}$ at $30 \mathrm{~Hz}$ ), due to the exponential loss of fractionated potentials (right lower panel). The relation between FDT and low-pass filtering was significant $(p<0.01)$.

\section{Impact of Notch Filtering on Morphology of Fibrillation Potentials}

As indicated in Table 2, notch filtering slightly decreased deflection amplitude and increased the percentage of CFPs, whereas no effect on FDT and the percentage of SPs and DPs was observed. Applying a notch filter at $50 \mathrm{~Hz}$ induced a loss of $\sim 1 \%$ detected fibrillation potentials (from $9234-14,545$ to $9234-14,405$ potentials, $p=0.01$ ). This significant, yet minimal, effect was also observed in a decrease in median deflection amplitude (from $0.59-0.96 \mathrm{mV}$ to $0.59-0.95 \mathrm{mV}, \mathrm{p}<0.01)$. Median FDT was not affected by notch filtering (from $16.0-25.0 \mathrm{~ms}$ to $16.0-25.0 \mathrm{~ms}, \mathrm{p}=$ NS). Notch filtering also had no effect on the percentage of SPs and DPs (from $36.1-57.6 \%$ to $35.8-57.6 \%$ and from $25.8-32.9 \%$ to $25.8-32.3 \%$ respectively, $p=\mathrm{NS}$ ), but did increase the percentage of CFPs significantly (from 15.9$36.0 \%$ to $16.2-37.0 \%, p=0.0157)$.

Table 2 Impact of notch filtering on unipolar fibrillation potential morphology. Delta values ( $\Delta=$ notch filter on-notch filter off) for all parameters and corresponding $p$ values based on the likelihood ratio test

\begin{tabular}{lllllll}
\hline Study ID & $\begin{array}{l}\Delta \text { Number of detected fibrillation } \\
\text { potentials }\end{array}$ & $\begin{array}{l}\Delta \text { Median deflection } \\
\text { amplitude }(\mathrm{mV})\end{array}$ & $\Delta$ Singles $(\%)$ & $\Delta$ Doubles $(\%)$ & $\Delta$ Complex $(\%)$ & $\begin{array}{l}\Delta \text { Median FDT } \\
(\mathrm{ms})\end{array}$ \\
\hline 1 & -42 & -0.0125 & 0.2728 & -0.5923 & 0.3196 & 0 \\
2 & -76 & -0.0131 & 0.1949 & -0.4636 & 0.2686 & 0 \\
3 & -132 & -0.0094 & 0.2474 & -0.1654 & -0.0820 & 0 \\
4 & 0 & 0.0000 & 0.0000 & 0.0000 & 0.0000 & 0 \\
5 & -213 & -0.0081 & -0.8585 & 0.4505 & 0.4080 & 0 \\
6 & -140 & -0.0050 & -0.3731 & 0.0758 & 0.2973 & 0 \\
7 & -61 & -0.0119 & -0.3039 & -0.6978 & 1.0017 & 0 \\
8 & 14 & -0.0163 & -0.4014 & 0.2530 & 0.1484 & -1 \\
9 & -11 & -0.0119 & 0.3255 & -0.4528 & 0.1100 & 0 \\
10 & -190 & -0.0081 & -0.1262 & 0.0469 & 0.0875 \\
Min-max & $-213-14$ & $-0.0163-0.0000$ & $-0.8585-0.3255$ & $-0.6978-0.4505$ & $-0.082-1.0017-1-0$ \\
$p$ value & $0.0043^{*}$ & $<0.0001 *$ & 0.3827 & 0.1973 & $0.0157^{*}$ & 0.3047 \\
\hline
\end{tabular}

\section{Filtering and Detection of Local Activation Time}

As a subanalysis, the impact of filtering on detection of LAT was determined. Results are described in detail in the ESM 3. All filter settings, i.e., all high-pass, low-pass, and notch filter settings, evoked changes in LAT timing (ESM 2). Especially with more aggressive low-pass filtering, the percentage of fibrillation potentials that had a shift in LAT was high (e.g., 58.32 to $63.12 \%$ at $100 \mathrm{~Hz}$ ). Filtering impacted LATs of all potential types (i.e., SPs, DPs, and CFPs). Nevertheless, more complex and long fractionated potentials had a greater $\Delta \mathrm{LAT}$ - and thus shifted more - than potentials with simpler morphology.

\section{Discussion}

All clinically used mapping systems - both unipolar and bipolar-standardly use signal filters while ablating. Although all mapping systems have different default filter settings, operators have the freedom and ability to change the filter settings according to their wishes. The results of our study clearly show that filtering choices have a significant impact on unipolar signal morphology. Attempts to correct for noise or baseline drift can therefore easily result in erroneous (under)detection of fractionation and/or low-voltage areas and thus ablative targets during mapping.

Our study thereby complements and verifies previously reported findings by Schneider et al. [21] and Lin et al. [22], in which unipolar endocardial peak-to-peak voltage decreased with increasing high-pass filtering in patients with ectopic atrial tachyarrhythmias and atrial flutter, respectively.

are given $(*<0.05)$. Potential type is either single (one deflection), double (two deflections), or complex (three or more deflections). FDT fractionation delay time 


\section{Current Clinical Use of Filtering in Ablative Techniques}

Although initially developed as a stand-alone strategy, ablation of tissue exhibiting CFAEs is nowadays generally used as adjuvant therapy to PV isolation, particularly in persistent $\mathrm{AF}$ patients $[2,23]$. Pathophysiological mechanisms of CFAE include pivoting points, inhomogeneous conduction, functional conduction block, reduced cell coupling, and interstitial fibrosis [2, 24]. On signal level, fractionation is often considered high-frequency content with a low-amplitude [25, 26]. Accordingly, lowering particularly the low-pass filter frequency impacted the presence of (complex) fractionated potentials in our study, since high-frequency content was eliminated. So while the decision to change the low-pass filter setting during CFAE ablation is probably made to reject high-frequency noise, detection of CFPs and thereby ablative targets is also strongly impeded. As an example, low-pass filtering at $100 \mathrm{~Hz}$ already eliminated up to $40 \%$ of fibrillation potentials and reduced the presence of CFPs from $15.9-36.0 \%$ to $2.3-$ $10.7 \%$ in all patients. Interestingly, increasing high-pass filtering did significantly decrease the percentage of fractionated potentials, primarily due to loss of complex fractionated potentials, as also observed in the decreasing FDT. In comparison to the impact of low-pass filtering however, this effect is clearly less substantial. On the contrary, notch filtering increased the presence of CFPs (max of $1.0017 \%$ increase) by adding artificial components to the unipolar fibrillation potential, just as in bipolar measurements [3].

Our results also stress the significance of adequate (i.e., as minimal as possible) filtering for voltage mapping, as unipolar potential amplitude was impacted by high-pass, low-pass, and notch filtering. Low-voltage areas $(<0.5 \mathrm{mV})$ have been linked to fibrosis, poor cell-to-cell coupling, slowed and discontinuous electrical conduction, and thus maintenance of $\mathrm{AF}$, motivating targeted ablation of these areas as an isolated approach or in addition to CFAE ablation and/or PV isolation [26, 27]. As our results indicate, low-pass filtering decreased the number of lowamplitude deflections, thereby reducing the number of potential target sites for low-voltage ablation in clinical practice. On the contrary, high-pass filtering attenuated the overall deflection amplitude and induced an increase in the number of low-amplitude signals, potentially leading to erroneous overdetection of lowvoltage target sites. Notch filtering did significantly lower deflection amplitude, but its impact is rather small $(-0.0163-$ $0.0000 \mathrm{mV}$ change in amplitude).

With increasing filtering (i.e., increasing high-pass or lowering low-pass cut-off frequencies), the number of detected fibrillation potentials declined. In addition, as described in the Supplemental material, filtering impacted timing of LAT. Though not specifically analyzed in this study, the missing potentials as well as the shift in LATs could lead to changes in detected activation patterns during AF. As such, inadequate filtering could result in unintentional under- or overestimation of rotational activity and focal, or peripheral, waves during mapping. A future study on the precise impact of filtering on measured activation sequences during $\mathrm{AF}$ could be very insightful.

As implied in the word itself, filtering inherently results in loss of information. Nevertheless, filtering does not have to be inaccurate if the lost information is irrelevant to your case. The challenge always lies in balancing minimal filtering with maximal signal quality (e.g., without artifacts and noise). However, since the relevant frequency content of fractionated fibrillation potentials is unknown and physiological discrimination between true fractionation and noise contribution is (yet) unfeasible, this study validates the use of minimal filtering. A safer alternative to filtering could be implementation of a signal-to-noise ratio (SNR) within mapping systems, in which detection criteria for fractionated and/or low-voltage potentials become stricter in case of considerable noise, without affecting original signal morphology. Using such an approach also prevents artifacts induced by filtering, such as ringing artifacts, to manifest.

Due to its ability to reduce far-field potentials by subtracting two unipolar electrograms at adjacent sites, bipolar electrograms are clinically often preferred above unipolar measurements. [11] For purposes of fractionation analyses, this favor is perhaps undeserved, since apart from filtering bipolar electrogram morphology is also susceptible to changes in interelectrode distance, electrode size and wavefront direction $[3,11,12,28]$

\section{The Pathophysiology of Fractionation}

The search for true - pathologic - fractionation remains an ongoing challenge. Differentiating between physiologic fractionation, due to, e.g., overlaying myocardial fibers and functional anisotropy, and pathologic fractionation for now remains difficult. The fact that measurement settings, such as filtering, affect fractionation as well provides another challenge. Nevertheless, the potential of fractionation-guided ablation could be significant if one could find methods for discerning true fractionation identifying abnormal conduction and arrhythmogenicity in AF patients. Specifically highresolution AF mapping studies, combined with imaging and/ or histologic techniques, could aid in unraveling the "fact or artifact" of fractionation.

\section{Limitations}

In this observational study, we included ten patients in whom we measured the impact of high-pass, low-pass, and notch filtering. Even though sample size seems rather small, a total of $3000 \mathrm{~s}$ of AF recordings, consisting of 2,557,045 fibrillation potentials were analyzed. Furthermore, our results 
indicate the impact of filtering to be very much alike between patients. Considering that filtering is a highly reproducible technique, we hypothesize that the general conclusions of this study can be extrapolated to each individual patient. Nevertheless, in clinical practice, there may be variations in filter properties. For example, to assure generalizability and reproducibility we used zero-phase filtering, but this might not be possible in daily clinical practice. For this study, we used unipolar intra-operative high-resolution mapping data, which is different from (bipolar) endocardial mapping data during ablative therapy with typically lower resolution data. For establishing the impact of filtering on unipolar data however, not spatial resolution but temporal resolution-thus sampling rate - is an important and relevant property, since filtering is done in time-domain. Clinically used mapping systems have comparable sampling rates of $\sim 1$ or $1.2 \mathrm{kHz}$, so our results should be considered relevant for endocardial unipolar mapping as well. Increasing sampling rate and resolution of analog to digital conversion and using wider band-pass filtering of origin could potentially further improve signal quality.

\section{Conclusions}

High-pass, low-pass, and notch filtering impacted morphology of unipolar fibrillation potentials, including amplitude, fractionation, and FDT, and decreased the number of detected fibrillation potentials, becoming a potential source of error in identification of low-voltage areas and (complex) fractionated potentials. While searching for ablative targets during clinical mapping, operators should be well aware of the consequences of filtering. In case of considerable noise, application of a signal-to-noise ratio - not affecting original signal morphology — could be a safer alternative.

Acknowledgments The authors would like to kindly thank F.B.S. Oei, $\mathrm{MD}, \mathrm{PhD}$; J.A. Bekkers, MD, PhD; P.C. van de Woestijne, MD; W.J. van Leeuwen, MD; Y.J.H.J. Taverne, MD, PhD; M.W.A. Bekker, MD; F.R.N. van Schaagen, MD; A. Yaksh, MD, PhD; E.M.J.P. Mouws, $\mathrm{MD}, \mathrm{PhD}$; C.P. Teuwen, MD; E.A.H. Lanters, MD; C.A. Houck, MD; L.J.M.E. van der Does, MD; A. Heida, MD; L.N. van Staveren, MD; W.F.B. van der Does, MD; C.S. Schram-Serban, DVM; R.K. Kharbanda, MD; and M.S. van Schie, MSc for their contribution to this work, as well as $\mathrm{H}$. Boersma MSc, PhD, FESC, for his advice regarding the statistical analysis of this study.

Funding Information N.M.S. de Groot, $\mathrm{MD}, \mathrm{PhD}$, is supported by grants from the Investigator-Initiated Study Program of Biosense Webster, Inc. (ISS-331 Phase 2), CVON-AFFIP (914728), NWO-Vidi (91717339) and Medical Delta.

\section{Compliance with Ethical Standards}

Conflict of Interest The authors declare that they have no conflict of interest.
Informed Consent All procedures followed were in accordance with the ethical standards of the responsible committee on human experimentation (institutional and national) and with the Helsinki declaration of 1975, as revised in 2000. Informed consent was obtained from all patients before inclusion in the study.

Open Access This article is licensed under a Creative Commons Attribution 4.0 International License, which permits use, sharing, adaptation, distribution and reproduction in any medium or format, as long as you give appropriate credit to the original author(s) and the source, provide a link to the Creative Commons licence, and indicate if changes were made. The images or other third party material in this article are included in the article's Creative Commons licence, unless indicated otherwise in a credit line to the material. If material is not included in the article's Creative Commons licence and your intended use is not permitted by statutory regulation or exceeds the permitted use, you will need to obtain permission directly from the copyright holder. To view a copy of this licence, visit http://creativecommons.org/licenses/by/4.0/.

\section{References}

1. Konings, K. T., Smeets, J. L., Penn, O. C., Wellens, H. J., \& Allessie, M. A. (1997). Configuration of unipolar atrial electrograms during electrically induced atrial fibrillation in humans. Circulation, 95(5), 1231-1241.

2. Nademanee, K., McKenzie, J., Kosar, E., Schwab, M., Sunsaneewitayakul, B., Vasavakul, T., et al. (2004). A new approach for catheter ablation of atrial fibrillation: Mapping of the electrophysiologic substrate. Journal of the American College of Cardiology, 43(11), 2044-2053. https://doi.org/10.1016/j.jacc. 2003.12.054

3. de Bakker, J. M., \& Wittkampf, F. H. (2010). The pathophysiologic basis of fractionated and complex electrograms and the impact of recording techniques on their detection and interpretation. Circulation. Arrhythmia and Electrophysiology, 3(2), 204-213. https://doi.org/10.1161/CIRCEP.109.904763.

4. van der Does, L. J., \& de Groot, N. M. (2017). Inhomogeneity and complexity in defining fractionated electrograms. Heart Rhythm, 14(4), 616-624. https://doi.org/10.1016/j.hrthm.2017.01.021.

5. Waxman, H. L., \& Sung, R. J. (1980). Significance of fragmented ventricular electrograms observed using intracardiac recording techniques in man. Circulation, 62(6), 1349-1356.

6. Klitzner, T. S., \& Stevenson, W. G. (1990). Effects of filtering on right ventricular electrograms recorded from endocardial catheters in humans. Pacing and Clinical Electrophysiology, 13(1), 69-77.

7. Verma, A., Jiang, C. Y., Betts, T. R., Chen, J., Deisenhofer, I., Mantovan, R., et al. (2015). Approaches to catheter ablation for persistent atrial fibrillation. The New England Journal of Medicine, 372(19), 1812-1822. https://doi.org/10.1056/ NEJMoa1408288.

8. Providencia, R., Lambiase, P. D., Srinivasan, N., Ganesh Babu, G., Bronis, K., Ahsan, S., et al. (2015). Is there still a role for complex fractionated atrial Electrogram ablation in addition to pulmonary vein isolation in patients with paroxysmal and persistent atrial fibrillation? Meta-analysis of 1415 patients. Circulation. Arrhythmia and Electrophysiology, 8(5), 1017-1029. https://doi.org/10.1161/ CIRCEP.115.003019.

9. Vogler, J., Willems, S., Sultan, A., Schreiber, D., Luker, J., Servatius, H., et al. (2015). Pulmonary vein isolation versus defragmentation: The CHASE-AF clinical trial. Journal of the American College of Cardiology, 66(24), 2743-2752. https://doi.org/10. 1016/j.jacc.2015.09.088. 
10. Seitz, J., Bars, C., Theodore, G., Beurtheret, S., Lellouche, N., Bremondy, M., et al. (2017). AF ablation guided by spatiotemporal Electrogram dispersion without pulmonary vein isolation: A wholly patient-tailored approach. Journal of the American College of Cardiology, 69(3), 303-321. https://doi.org/10.1016/j.jacc.2016. 10.065 .

11. Venkatachalam, K. L., Herbrandson, J. E., \& Asirvatham, S. J. (2011). Signals and signal processing for the electrophysiologist: Part II: Signal processing and artifact. Circulation. Arrhythmia and Electrophysiology, 4(6), 974-981. https://doi.org/10.1161/ CIRCEP.111.964973.

12. Zaman, J. A. B., Schricker, A., Lalani, G. G., Trikha, R., Krummen, D. E., \& Narayan, S. M. (2014). Focal impulse and rotor mapping (FIRM): Conceptualizing and treating atrial fibrillation. Journal of Atrial Fibrillation, 7(2), 1103. https://doi.org/10.4022/jafib.1103.

13. Grace, A., Willems, S., Meyer, C., Verma, A., Heck, P., Zhu, M., et al. (2019). High-resolution noncontact charge-density mapping of endocardial activation. JCI Insight, 4(6), e126422. https://doi. org/10.1172/jci.insight.126422.

14. van der Does, L. J. M. E., Knops, P., Teuwen, C. P., Serban, C., Starreveld, R., Lanters, E. A. H., et al. (2018). Unipolar atrial electrogram morphology from an epicardial and endocardial perspective. Heart Rhythm, 15(6), 879-887. https://doi.org/10.1016/j. hrthm.2018.02.020.

15. Lanters, E. A., van Marion, D. M., Kik, C., Steen, H., Bogers, A. J., Allessie, M. A., et al. (2015). HALT \& REVERSE: Hsfl activators lower cardiomyocyt damage; towards a novel approach to REVERSE atrial fibrillation. Journal of Translational Medicine, 13, 347. https://doi.org/10.1186/s12967-015-0714-7.

16. van der Does, L. J., Yaksh, A., Kik, C., Knops, P., Lanters, E. A., Teuwen, C. P., et al. (2016). QUest for the Arrhythmogenic substrate of atrial fibRillation in patients undergoing cardiac surgery (QUASAR study): Rationale and design. Journal of Cardiovascular Translational Research, 9(3), 194-201. https:// doi.org/10.1007/s12265-016-9685-1.

17. Teuwen, C. P., Yaksh, A., Lanters, E. A., Kik, C., van der Does, L. J., Knops, P., et al. (2016). Relevance of conduction disorders in Bachmann's bundle during sinus rhythm in humans. Circulation. Arrhythmia and Electrophysiology, 9(5), e003972. https://oi.org/ 10.1161/CIRCEP.115.003972.

18. Mouws, E., Lanters, E. A. H., Teuwen, C. P., van der Does, L., Kik, C., Knops, P., et al. (2017). Epicardial breakthrough waves during sinus rhythm: Depiction of the Arrhythmogenic substrate? Circulation. Arrhythmia and Electrophysiology, 10(9), e005145. https://doi.org/10.1161/CIRCEP.117.005145.

19. Kik, C., Mouws, E., Bogers, A., \& de Groot, N. M. S. (2017). Intraoperative mapping of the atria: The first step towards individualization of atrial fibrillation therapy? Expert Review of Cardiovascular Therapy, 15(7), 537-545. https://doi.org/10.1080/ 14779072.2017.1340156.

20. de Groot, N. M., Houben, R. P., Smeets, J. L., Boersma, E., Schotten, U., Schalij, M. J., et al. (2010). Electropathological substrate of longstanding persistent atrial fibrillation in patients with structural heart disease: Epicardial breakthrough. Circulation, 122(17), 1674-1682. https://doi.org/10.1161/ CIRCULATIONAHA.109.910901.

21. Schneider, M. A., Ndrepepa, G., Weber, S., Deisenhofer, I., Schomig, A., \& Schmitt, C. (2004). Influence of high-pass filtering on noncontact mapping and ablation of atrial tachycardias. Pacing and Clinical Electrophysiology, 27(1), 38-46. https://doi.org/10. 1111/j.1540-8159.2004.00383.x.

22. Lin, Y. J., Tai, C. T., Lo, L. W., Udyavar, A. R., Chang, S. L., Wongcharoen, W., et al. (2007). Optimal electrogram voltage recording technique for detecting the acute ablative tissue injury in the human right atrium. Journal of Cardiovascular Electrophysiology, 18(6), 617-622. https://doi.org/10.1111/j.1540-8167.2007.00803. $\mathrm{x}$.

23. Wu, S. H., Jiang, W. F., Gu, J., Zhao, L., Wang, Y. L., Liu, Y. G., et al. (2013). Benefits and risks of additional ablation of complex fractionated atrial electrograms for patients with atrial fibrillation: A systematic review and meta-analysis. International Journal of Cardiology, 169(1), 35-43. https://doi.org/10.1016/j.ijcard.2013. 08.083.

24. Konings, K. T., Kirchhof, C. J., Smeets, J. R., Wellens, H. J., Penn, O. C., \& Allessie, M. A. (1994). High-density mapping of electrically induced atrial fibrillation in humans. Circulation, 89(4), $1665-1680$.

25. Stiles, M. K., Brooks, A. G., Kuklik, P., John, B., Dimitri, H., Lau, D. H., et al. (2008). High-density mapping of atrial fibrillation in humans: Relationship between high-frequency activation and electrogram fractionation. Journal of Cardiovascular Electrophysiology, 19(12), 1245-1253. https://doi.org/10.1111/j. 1540-8167.2008.01253.x.

26. Jadidi, A. S., Lehrmann, H., Keyl, C., Sorrel, J., Markstein, V., Minners, J., et al. (2016). Ablation of persistent atrial fibrillation targeting low-voltage areas with selective activation characteristics. Circulation. Arrhythmia and Electrophysiology, 9(3), e002962. https://doi.org/10.1161/CIRCEP.115.002962.

27. Blandino, A., Bianchi, F., Grossi, S., Biondi-Zoccai, G., Conte, M. R., Gaido, L., et al. (2017). Left atrial substrate modification targeting low-voltage areas for catheter ablation of atrial fibrillation: A systematic review and meta-analysis. Pacing and Clinical Electrophysiology, 40(2), 199-212. https://doi.org/10.1111/pace. 13015 .

28. Stevenson, W. G., \& Soejima, K. (2005). Recording techniques for clinical electrophysiology. Journal of Cardiovascular Electrophysiology, 16(9), 1017-1022. https://doi.org/10.1111/j. 1540-8167.2005.50155.x.

Publisher's Note Springer Nature remains neutral with regard to jurisdictional claims in published maps and institutional affiliations. 